Quantum Dot Sensitized Solar Cells

\title{
Turn Defects into Strengths
}

Iván Mora-Seró

\section{The power conversion efficiency of solar cells sensitized with colloidal quantum dots is believed to be limited by surface defects. Research now finds that photocarriers trapped at shallow defect states can actually be recovered and ultimately contribute to device efficiency.}

Quantum dots (QDs) are promising materials for photovoltaic applications owing to a variety of advantages over their bulk conterparts. ${ }^{1}$ For instance, the bandgap of QDs can be easily tuned by varying their size, which make them attractive for multiplejunction devices. Meanwhile, in QDs carrier cooling and charge recombination rates are slowed down, making them particularly interesting for third generation solar cells based on hot carrier effects and multiple electron-hole pair generation. ${ }^{1}$

QDs can be exploited as absorber in solar cells using two device architectures: a thinfilm QD solar cell or a QD sensitized solar cell (QDSSC). In thin-film devices, a layer of QDs (on the order of hundreds of microns) is sandwiched between two electrodes. The relatively thick layer allows for efficient light absorption. However, carrier transport in thin-film QD cells is hindered by the presence of ligands between QDs, trap states and broad QD size distribution (which introduces a broad distribution of bandgaps). The QDSSC configuration - where a monolayer attached to a mesoporous electrode and interfaced with a liquid electrolyte - instead ensures an efficient charge transport as charges photogenerated in the QDs are directly injected into the transporting materials. ${ }^{2,3}$ Yet, while QDSSC efficiency is similar to that of dyesensitized solar cells, ${ }^{3}$ it still lags behind that of thin-film QD and bulk solar cells as photocurrent and photovoltage need to be further improved. 
Researchers have traditionally focussed on two factors to maximise device efficiency: the elimination of surface defects by terminating QDs with surface passivation; and strengthening the coupling between the QDs and the electron transporting material (ETM) to ensure efficient charge injection and QD loading. Writing in Nature Energy, Victor Klimov and colleagues from Los Alamos National Laboratory in the USA confirm that the density of QDs on the ETM is a key factor in determining QDSSC efficiency while reporting that trapped photogenerated carriers can be partially recovered and consequently recycled to ultimately contribute to the device efficiency. ${ }^{4}$

Klimov and colleagues investigate $\mathrm{Zn}: \mathrm{CuInSe} 2 \mathrm{QDs}$, which typically enable high efficiencies in QDSSCs. ${ }^{3,5}$ This material composition is also particularly interesting as it avoids the use of toxic elements such as $\mathrm{Pb}$ or $\mathrm{Cd}$ typically employed in QD solar cells. The length of the organic ligand side chain regulates the steric hindrance of QDs and therefore their loading density. The ligand binding mode, instead, controls the coupling of the $\mathrm{QD}$ to the $\mathrm{TiO}_{2}$-based ETM. The researchers vary these two parameters systematically to investigate the impact of QD density, coupling strength and defect states on the charge carrier dynamics (Figure 1a).

They provide spectroscopic evidence that electrons trapped into intragap defect states are injected into the ETM when such defects are located above the $\mathrm{TiO}_{2}$ conduction band (Figure 1b, process 3). The same process occurs for holes trapped at defects formed below the redox level of the electrolyte (Figure 1b, process 7) which are nevertheless injected into the HTM. This observation suggests that these shallow defects assist charge transport and hence contribute to device efficiency rather than being detrimental. The need for a strong QD-ETL coupling, which is typically pursued to obtain photocarrier injection faster than charge trapping, and an efficient surface defect passivation are therefore not as crucial as previously thought to charge transport in QDSSC configuration, in comparison with the QD loading. High QD loading enhances light harvesting and, as a consequence, the photocurrent output. These findings indicate that future strategies to increase QDSSC performance should focus mostly on the increase of the QD loading rather than on the surface passivation and QD/ETL coupling due to the harnessing of trapped carriers.

Klimov and colleagues also discuss the origin of device instability in archetypal QDSSCs. They show that the polysulfide redox system normally used as the electrolyte 
in QDSSCs promotes the oxidation of the QDs and the oxidation of $\mathrm{Cu}$ in the brass - a $\mathrm{Cu}$ and $\mathrm{Zn}$ alloy - electrode. These oxidation processes limit both the open circuit potential and the long-term stability of the device. The researchers show that higher $\mathrm{V}_{\mathrm{oc}}$ and power conversion efficiency can be obtained using porous carbon counter electrodes. Nevertheless, this choice is not optimal in terms of long-term stability as the large surface area of the carbon material promotes the fast oxidation of the polysulfide redox. These observations provide valuable insights into the degradation mechanisms of QDSSCs which have been overlooked in the past. It becomes clear that alternative redox systems and counter electrodes must be developed to improve the reliability of QDSSCs.

With their study, Klimov and colleagues confirm the role of QD loading and reporting the trapped photocarrier recycling. The work also highlights the importance of electrolyte and counter electrode compatibility for improving device performance, and especially device stability. These findings could eventually lead to further gains in the device efficiency and stability of QDSSCs consolidating the potential and reliability of technology.
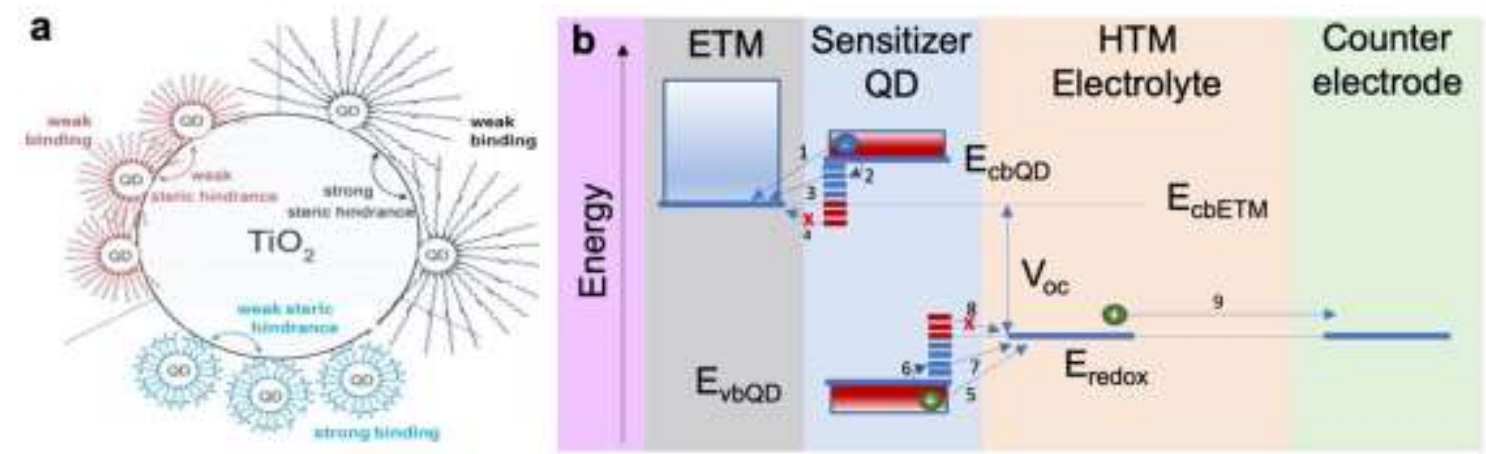

Figure 1: Working principle of defect-mediated charge injection in quantum dot sensitized solar cells. (a) Schematic diagram showing the QDs with ligands different in terms of length of the organic ligand side chain and ligand binding mode as investigated by Klimov and colleagues. Long-chain ligands result in a strong steric hindrance and therefore low QDs loading density (case illustrated in black). Ligands with specific functional groups enable a strong couple between QDs and $\mathrm{TiO}_{2}$ (case illustrated in blue). (b) In a QDSSC light is harvested by the QD sensitizer, which quickly injects the photogenerated carriers into the electron and hole transporting materials, ETM and HTM respectively. Electrons photoexcited to the conduction band of the QD, $\mathrm{E}_{\mathrm{cb}} \mathrm{QD}$, are injected into the conduction band of the ETM, $\mathrm{E}_{\mathrm{cb}}$ ETM (process 1). Analogously, photogenerated holes in the valence band of the QD, $\mathrm{E}_{\mathrm{vb}} \mathrm{QD}$, are injected into the redox level of the HTM, Eredox (process 5). Both electrons and holes can be trapped at QD surface defect states (processes 2 and 6 respectively). If carriers are trapped into shallow 


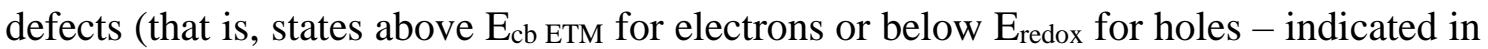
blue) they can be recovered and contribute to the device efficiency (processes 3 and 7, respectively). Electrons and holes trapped into deeper states (below $\mathrm{E}_{\mathrm{cb}}$ ETM for electrons or above $E_{\text {redox }}$ for holes - indicated in red) cannot be extracted (processes 4 and 8 respectively). Figure 1a is adapted from ref. 4.

\author{
Affiliation \\ Institute of Advanced Materials (INAM), Universitat Jaume I (UJI), Castelló de la \\ Plana, Spain. \\ sero@uji.es
}

\title{
References
}

1 Lu, H. et al. Energy Environ. Sci., doi:10.1039/C9EE03930A (2020).

2 O' Regan, B. \& Grätzel, M. Nature 353, 737-740 (1991).

3 Pan, Z. et al. Chem. Soc. Rev. 47, 7659-7702, (2018).

$4 \quad$ Du et. al Nat. Energy (2020)

5 Wang, W. et al. J. Am. Chem. Soc. 141, 4300-4307, (2019). 